\title{
The Small Exoplanet Mass-Radius Relation: Quantifying the Astrophysical Scatter
}

\author{
Angie Wolfgang ${ }^{1}$, Leslie A. Rogers ${ }^{2}$ and Eric B. Ford ${ }^{3}$ \\ ${ }^{1}$ NSF Postdoctoral Fellow, Dept. of Astronomy \& Astrophysics, Penn State University \\ ${ }^{2}$ Sagan Fellow, Dept. of Astronomy, University of California, Berkeley \\ ${ }^{3}$ Dept. of Astronomy \& Astrophysics; Center for Astrostatistics, Penn State University
}

\begin{abstract}
The Kepler Mission has discovered thousands of planets with radii $<4 \mathrm{R}_{\oplus}$, paving the way for the first statistical studies of super-Earth dynamics, formation, and evolution. These calculations often require planetary masses, and yet the vast majority of Kepler planet candidates do not have theirs measured. A key concern is therefore how to map the measured radii to mass estimates in a size range that lacks Solar System analogs. While previous works have derived one-to-one relationships between radius and mass, a realistic mass-radius (M-R) relation should account for the range of compositions that we expect within the population. This compositional diversity creates astrophysical scatter in the relation, which we quantify here.
\end{abstract}

We have applied a hierarchical Bayesian model to quantify the intrinsic population dispersion in the small planet M-R relation, in the spirit of Kelly (2007). This method has several added benefits over other techniques, including: effortless inclusion of large mass uncertainties and upper limits; self-consistent incorporation of uncertainties on the independent variable — radii in this case — without the need for elaborate bootstrapping schemes; and the production of posterior distributions, allowing easy characterization of the uncertainties in both the population-wide and planet-specific parameters.

In Wolfgang, Rogers, \& Ford (2015), we analyze how the constraints on this relation depend on the radius range of the sample, and on the method used to provide the mass measurements. Assuming that the M-R relation can be described as a power law with a dispersion that is constant and normally distributed, we find that $M / M_{\oplus}=2.7\left(R / R_{\oplus}\right)^{1.3}$ is the best fit "average" relation for the sample of RV-measured transiting sub-Neptunes $\left(R_{p l}<4 \mathrm{R}_{\oplus}\right)$, and that the astrophysical scatter around this "average" relation is $1.9 M_{\oplus}$.

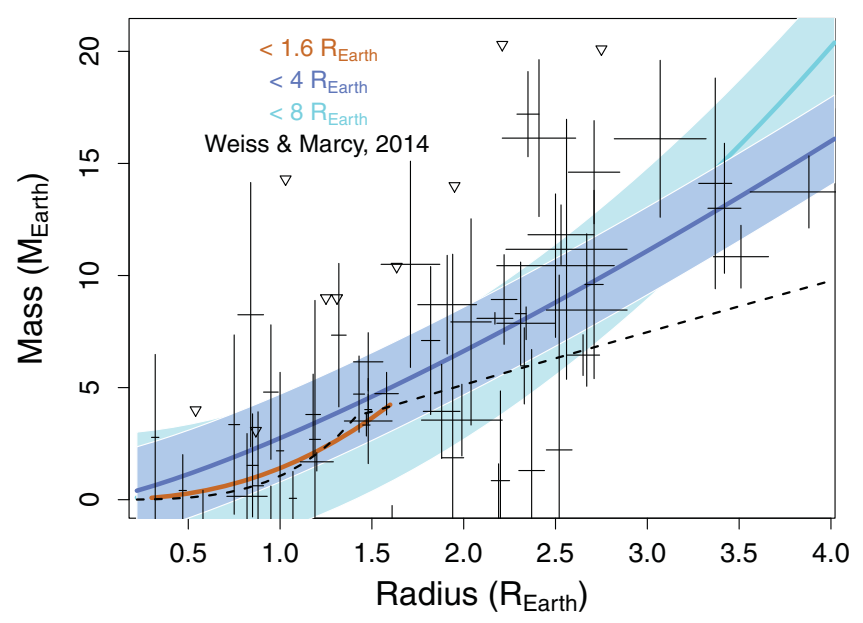

Figure 1. The best-fit M-R relations for data sets spanning different ranges of planetary radii. For each, the solid line denotes the "average" relation while the faded region denotes the standard deviation of the intrinsic, astrophysical scatter (note there is not yet sufficient observational evidence for scatter among the smallest planets). The dataset is overplotted as the thin black lines with triangles for the upper limits; a commonly used one-to-one relation is the dotted black line. 